\title{
Efficacy and safety of daclatasvir plus asunaprevir for Korean patients with HCV genotype lb infection: a retrospective multi-institutional study
}

\author{
Byeong Wook Cho', Seok Bae Kim, Il Han Song', Sae Hwan Lee², Hong Soo Kim², Tae Hee Lee ${ }^{3}$, Young Woo Kang ${ }^{3}$, \\ Seok Hyun Kim${ }^{4}$, Byung Seok Lee ${ }^{4}$, and Hee Bok Chae ${ }^{5}$ \\ 'Department of Internal Medicine, Dankook University College of Medicine; ${ }^{2}$ Department of Internal Medicine, Soonchunhyang \\ University College of Medicine, Cheonan; ${ }^{3}$ Department of Internal Medicine, Konyang University College of Medicine; ${ }^{4}$ Department of \\ Internal Medicine, Chungnam University College of Medicine, Daejeon; ${ }^{5}$ Department of Internal Medicine, Chungbuk University College \\ of Medicine, Cheongju, Korea
}

Background/Aims: The combination of daclatasvir (DCV) and asunaprevir (ASV) has demonstrated a high sustained virologic response at 12 weeks (SVR12) and a low rate of adverse events in previous clinical studies. The purpose of this study was to clarify the results of treatment and side effects in Korean patients with chronic hepatitis C virus (HCV) genotype lb infection.

Methods: We retrospectively analyzed clinical data from chronic HCV genotype lb patients treated with DCV+ASV from August 2015 to September 2016 at five hospitals in the Daejeon-Chungcheong area.

Results: A total of 152 patients were examined for resistance associated variants (RAVs). Among them, 15 (9.9\%) were positive for $Y 93$ and one (0.7\%) was positive for L31. Of 126 patients treated with DCV+ASV, 83 patients completed treatment and 76 patients were included in safety and efficacy analysis. Five (6.6\%) were positive for Y93 and 12 (15.8\%) exhibited cirrhotic change. DCV+ASV was the first-line treatment for 58 (76.3\%) patients. Eleven (14.5\%) patients relapsed after previous treatment that included interferon and seven (9.2\%) of these patients were found to be intolerant of interferon. Adverse events occurred in $10(13.2 \%)$ patients and two patients stopped the medication because of severe itching and skin rash. SVR12 was 89.5\% (68/76) in all patients and 91.5\% (65/71) in RAV-negative patients.

Conclusions: DCV+ASV showed good efficacy in patients with HCV Ib infection in Korea. Close monitoring is needed for severe adverse events and treatment failure, which were uncommon. (Clin Mol Hepatol 2017;23:51-56)

Keywords: Daclatasvir; Asunaprevir; Hepatitis C, Chronic; Korea

\section{INTRODUCTION}

Chronic hepatitis C virus (HCV) infection was once best treated using peginterferon plus ribavirin. The difficulties with this treat- ment included severe adverse events, inconvenience of injection, and the long treatment duration required. The cure rate was only $60-70 \%$, although it has improved.

This treatment was supplanted by use of direct acting antivirals

\section{Abbreviations:}

ASV, asunaprevir; DAAs, direct acting antivirals; DCV, daclatasvir; ETR, end of treatment response; $\mathrm{HCV}$, hepatitis $\mathrm{C}$ virus; LLOQ, lower limit of quantification; NS5A, non-structural 5A; RAV, resistance associated variant; SVR12, sustained virologic response at 12 weeks

\section{Corresponding author : Seok Bae Kim}

Department of Internal Medicine, Dankook University College of Medicine, 119 Dandae-ro, Dongnam-gu, Cheonan 31116, Korea

Tel: +82-41-550-3910, Fax: +82-41-556-3256

E-mail:dryakson@hanmail.net 
(DAAs) in the mid-1990s. The cure rate increased to over 90\%, the treatment duration was shortened, and treatment became more convenient since an injection was not required. Peginterferon is no longer recommended as the first-line treatment. ${ }^{2-4}$

The combination of daclatasvir (DCV) and asunaprevir (ASV) was developed in Japan for the treatment of HCV genotype Ib. A few clinical studies including some Korean patients reported the high sustained virologic response at 12 weeks (SVR12) rate and a low rate of adverse events. The DCV+ASV combination was the first DAA approved for use in Korea in August 2015 with some usage limitations. This study aimed to clarify the results of treatment and side effects in Korean patients with chronic HCV genotype $\mathrm{lb}$ infection treated with DCV+ASV.

\section{MATERIALS AND METHODS}

\section{Patients}

We included the patients who was infected with HCV genotype $\mathrm{Ib}$ and took examination for resistance associated variants (RAV) at 5 university hospitals located on Daejeon-Chungcheong area. The patients with decompensated cirrhosis were excluded by calculating Child-Pugh score with abdominal sonography, computed tomography, and blood test. The enrolled patients were divided into the treatment naïve group, treatment failure group (including null response, partial response, virologic breakthrough, relapse to previous treatment), and intolerant group (discontinued peginterferon because of side effects). The definition of virologic response followed the Korean Association for the Study of the Liver (KASL) guideline. ${ }^{3}$ Positive RAV rate was determined in all enrolled patients. Rates of end of treatment response (ETR), SVR12, adverse events were analyzed in patients who completed the treatment. The protocol was approved by the institutional review board or independent ethics committee at each site. Patients were not required to give informed consent because this study used clinical data obtained after patients had agreed to treatment before initiation of treatment.

\section{RAV examination}

RAV examination focused on variants of non-structural $5 \mathrm{~A}$ (NS5A). All five sites submitted samples for testing at Seoul Medical Science Institute. NS5A was detected by direct sequencing using a Veriti 96 well thermal cycler and genetic analyzer (Applied
Biosystems, Carlsbad, CA, USA). The limit of detection of HCV RNA was 5,000 IU/mL. The result was recorded as positive or negative according to presence of RAV sites L31 or Y93. If result was "not detected", reexamination of HCV genotype was done to exclude the other genotype.

\section{Clinical and laboratory assessments}

Hemotological and biochemical tests were performed before and during treatment at as close as possible to 4, 8, 16, 24 weeks during and 12 weeks after treatment. Cirrhosis of the liver was diagnosed by abdominal sonography and computed tomography. The Child-Pugh score was calculated to determine the difference in treatment results. Adverse events were identified by review of medical records and were confirmed concerning onset time, severity, and duration by discussion with co-authors at each site.

\section{Measurement of HCV RNA and assessment of treatment efficacy}

HCV RNA was measured by a real-time PCR assay at each site. The lower limit of quantification (LLOQ) of HCV RNA was $15 \mathrm{lU} / \mathrm{mL}$ at four sites and $40 \mathrm{IU} / \mathrm{mL}$ at the other site. Virologic breakthrough was defined as an increase of HCV RNA $>1 \log _{10}$ from nadir or HCV RNA $\geq$ LLOQ after a measurements below LLOQ. ETR was defined as undetectable HCV RNA at the end of 24 weeks of treatment and SVR12 was defined as undetectable HCV RNA at 12 weeks after treatment.

\section{Statistical analyses}

Data were expressed as mean \pm standard deviation. Chi-square test or Student's $t$-test were for univariate analyses. $P<0.05$ was considered statistically significant.

\section{RESULTS}

\section{Prevalence of RAV}

Total 152 patients comprised of 62 (40.8\%) men and 90 (59.2\%) women took RAV examination. Their mean age was $55.8 \pm 11.7$ years old. Fifteen (9.9\%) patients and one $(0.7 \%)$ patient showed positive RAV at Y93 and L31, respectively. No patient showed positive RAV at both regions. Six (9.7\%) men and 10 
(11.1\%) women showed positive RAV with no significant difference. The prevalence of RAV was 10.6\% (Table 1).

\section{Baseline characteristics of the treated patients}

Of 126 patients treated with DCV+ASV, 83 patients finished the treatment. Seven patients were excluded due to follow-up loss $(n=5)$, HCC recurrence $(n=1)$ and expire because of accident $(n=1)$. Finally, 76 patients including 32 (42.1\%) men and 44 (57.9\%) women were analyzed for safety and efficacy (Fig. 1). Mean age was 54.7 \pm 10.9

Table 1. Baseline characteristics of the patients who underwent RAV examination

\begin{tabular}{|cc}
\hline Variable & Value \\
\hline Total & 152 \\
\hline Male & $62(40.8)$ \\
\hline Female & $90(59.2)$ \\
\hline Age & $55.8 \pm 11.7$ \\
\hline RAV & \\
\hline L31 & $1(0.7)$ \\
\hline Y93 & $15(9.9)$ \\
\hline Male & $6 / 62(9.7)$ \\
\hline Female & $10 / 90(11.1)$ \\
\hline Total & $16(10.6)$ \\
\hline
\end{tabular}

Values are presented as mean \pm SD or $n(\%)$.

SD, standard deviation; RAV, resistance associated variants.

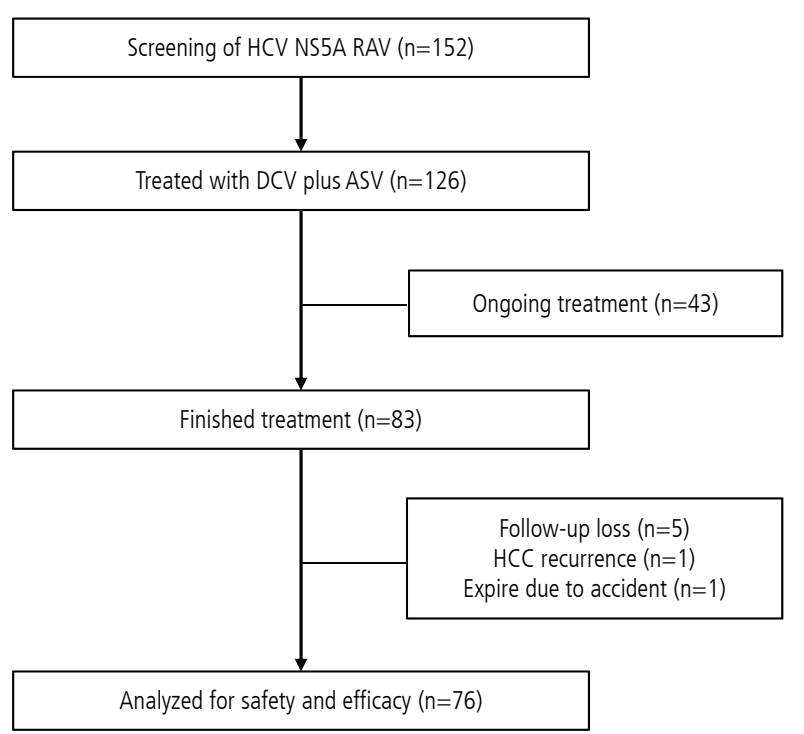

Figure 1. Flow chart summarizing patient selection. $H C V$, hepatitis $C$ virus; NS5A, non-structural 5A; RAV, resistance-associated variant; DCV, daclatasvir; ASV, asunaprevir. years. Five (6.6\%) patients who showed positive RAV (Y93) were included. Compensated cirrhosis was present at baseline in 14 $(18.4 \%)$ patients and absent in $62(81.6 \%)$ patients. There were 58 (76.3\%) treatment-naïve patients, $11(14.5 \%)$ treatment failure patients, and 7 (9.2\%) patients who did not tolerate peginterferon+ribavirin treatment (Table 2).

\section{Adverse event}

Adverse treatment-related events were reported in $10(13.2 \%)$

Table 2. Baseline characteristics of patients treated with DCV+ASV

\begin{tabular}{|c|c|}
\hline Variable & Value \\
\hline Total & 76 \\
\hline Male & $32(42.1)$ \\
\hline Female & $44(57.9)$ \\
\hline Age & $54.7 \pm 10.9$ \\
\hline \multicolumn{2}{|l|}{ RAV } \\
\hline L31 & $0(0)$ \\
\hline Y93 & $5(6.6)$ \\
\hline LC & $14(18.4)$ \\
\hline Naive & $58(76.3)$ \\
\hline Treatment failure & $11(14.5)$ \\
\hline Intolerant to PEG-IFN & $7(9.2)$ \\
\hline Mean HCV RNA value $\left(\log _{10} \mathrm{IU} / \mathrm{mL}\right)$ & $6.5 \pm 6.6$ \\
\hline Mean ALT level (IU/mL) & $55.9 \pm 58.6$ \\
\hline
\end{tabular}

Values are presented as mean \pm SD or $n(\%)$.

SD, standard deviation; RAV, resistance associated variants; LC, liver cirrhosis; PEG-IFN, peginterferon; ALT, alanine aminotransferase.

Table 3. Summary of adverse events in patients treated with DCV+ASV (76 patients)

\begin{tabular}{ll}
\hline Event & Value \\
\hline Liver enzyme elevation & $2(2.6)$ \\
Gl problem & $4(5.2)$ \\
\hline Anorexia & 1 \\
\hline Abdominal pain & 1 \\
\hline Indigestion & 2 \\
\hline Skin problem & $4(5.2)$ \\
\hline Skin rash & 3 \\
\hline Itching & 2 \\
\hline Generalized weakness & $2(2.6)$ \\
\hline Total & $10(13.2)$ \\
\hline
\end{tabular}

Values are presented as $n(\%)$. Two patients complained of two kinds of symptoms.

$\mathrm{Gl}$, gastrointestinal. 
patients. Transient aminotransferase increase $<3$-fold was noted in two (2.6\%) patients. Four (5.2\%) patients complained of gastrointestinal discomfort including anorexia $(n=1)$, abdominal pain $(n=1)$, and indigestion ( $n=2$ ) without discontinuing treatment. Four (5.2\%) patients suffered from skin rash and itching. Among them, two patients stopped the drugs due to severe itching. One of the two patients was a 59 year old female who relapsed after peginterferon+ribavirin treatment. She stopped treatment after 8 days. The second patient was an 80 year old female who had no previous treatment history. She first stopped the drugs at 20 days after treatment and restarted again after several days. But she finally stopped after 4 months because of recurrence of severe itching and rash. HCV RNA was not detected at 12 weeks after treatment (Table 3).

\section{Virologic response}

Seventy-six patients were analyzed for virologic response. SVR12 was $89.5 \%(68 / 76)$ overall, $89.7 \%(52 / 58)$ in the treatment-naïve group, $81.8 \%(9 / 11)$ in the treatment-failure group, and $100 \%(7 / 7)$ in the treatment-intolerant group. SVR12 for negative RAV patients was $91.5 \%$ (65/71) overall, 90.9\% (50/55) in the treatment-naive group, $88.9 \%(8 / 9)$ in the treatment-failure group, and $100 \%(7 / 7)$ in the treatment-intolerant group (Fig. 2).

Sixty nine $(90.8 \%)$ patients achieved ETR. The one patient displayed detectable RNA at 24 weeks after treatment. Y93 was positive on pretreatment examination of the patient. One patient who achieved ETR showed detectable HCV RNA at 12 weeks af-

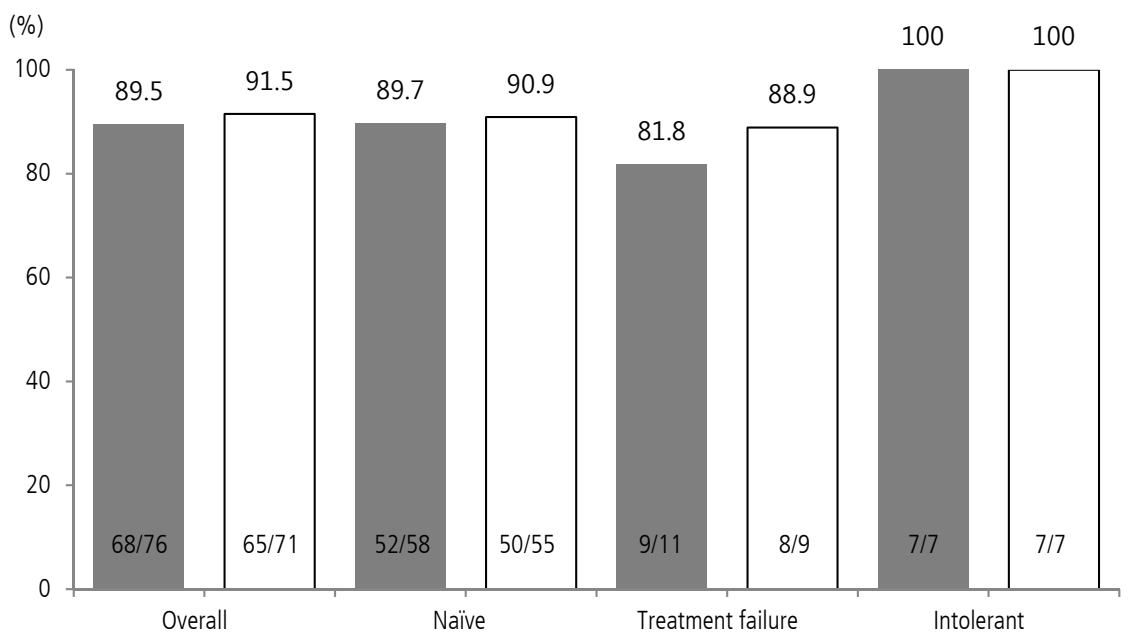

Figure 2. SVR12 overall and by prior treatment status in patients treated with DCV+ASV analyzed according to the presence of RAV. SVR12 was higher in $\operatorname{RAV}(-)$ patients than in $\mathrm{RAV}(+)$ patients. DCV, daclatasvir; ASV, asunaprevir; RAV, resistance-associated variant; SVR12, sustained virologic response 12 weeks post-treatment.

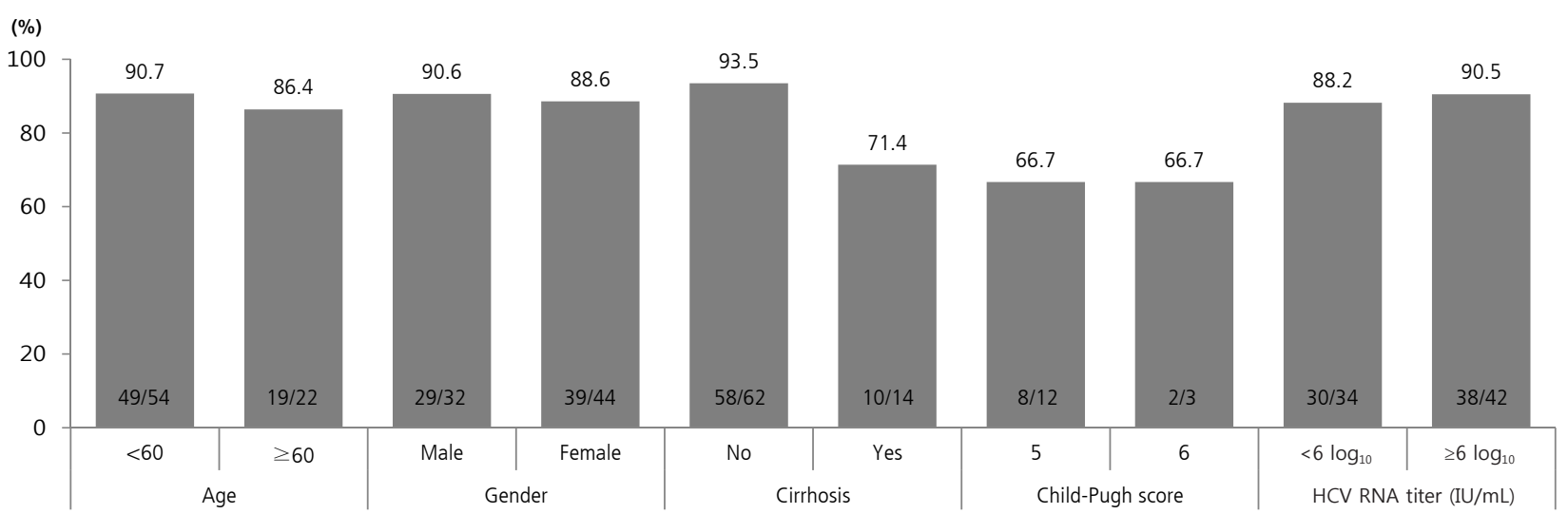

Figure 3. SVR12 according to baseline age, sex, presence of cirrhosis, Child-Pugh score, and pretreatment HCV RNA level. There was a higher SVR12 tendency in those who were male, younger than 60 years, without cirrhosis, and with an HCV RNA level $\geq 6 \log 10 \mathrm{lU} / \mathrm{mL}$, but these findings were not statistically significant. HCV, hepatitis C virus; SVR12, sustained virologic response 12 weeks post-treatment. 
Byeong Wook Cho, et al.

Efficacy of daclatasvir plus asunaprevir

ter cessation of treatment. The patient was relapsed after 48 weeks treatment of peginterferon+ribavirin and showed positive Y93.

SVR12 was analyzed according to baseline factors of age, sex, presence of cirrhosis, Child-Pugh score, and HCV RNA level. There was higher SVR12 tendency in patients younger than 60 years, in male, without cirrhosis, and HCV RNA level $\geq 6 \log _{10} \mathrm{IU} / \mathrm{mL}$ without statistical significance (Fig. 3).

\section{DISCUSSION}

DCV is a NS5A inhibitor that displays antiviral activity to all HCV genotypes in vitro. ${ }^{5}$ ASV is a NS3 protease inhibitor that has antiviral activity to HCV genotypes 1, 4, 5, and 6 in vitro. ${ }^{6}$ The authors of the study conducted in Japan reported that DCV+ASV produced high and low SVR to HCV genotype $\mathrm{Ib}$ and la, respectively. Use of DCV+ASV only for HCV genotype Ib in Japan was recommended and implemented. Insurance coverage of these drugs in Korea in effect since August 2015 limited against genotype $\mathrm{Ib}^{7}$

This genotype is the most prevalent subtype in Korea, accounting for a reported $45.4 \%$ of cases of $\mathrm{HCV}^{8}{ }^{8} \mathrm{DCV}+\mathrm{ASV}$ have been widely prescribed for treatment of chronic HCV infection in Korea, given the aforementioned findings and insurance coverage. The higher SVR of DCV+ASV compared with peginterferon+ribavirin is an advantage. Use of DCV+ASV produced SVR12 in $83.7 \%$ of treated Asian patients in a clinical study. ${ }^{9}$ Subgroup analysis in the same study revealed SVR12 rates of was $92.3 \%$ in the treatmentnaïve group, $80 \%$ in the prior non-responders group, and $78.6 \%$ in the ineligible/intolerant group. Previous treatment history was a predictive factor for SVR12 in this prior and the present studies. Presently, the SVR12 rate of $89.7 \%$ in the treatment-naïve group was higher than the rate in the treatment failure group (81.8\%), similar to a prior clinical study. ${ }^{10}$

The presence of RAV was most important predictive factor of SVR in DCV+ASV treatment. Cases featuring positive RAV in a pretreatment examination are not eligible for insurance coverage in Korea. The prevalence of RAV is differs according to race and country; Japan has highest prevalence of RAV and non-Asian countries have lower prevalence of RAV than Asian countries. ${ }^{11-13}$ The HALLMARK DUAL study, which included 78 Korean patients and 85 Taiwanese patients, reported an $11.8 \%$ (18/153) prevalence of RAV. ${ }^{9}$ Among the 18 patients, 15 were $Y 93$ positive and the remaining three were $\mathrm{L} 31$ positive. In the present study, 152 patients were examined for RAV; the resulting prevalence of $10.6 \%$ was similar with the prior study.

Presently, SVR12 in cases of positive RAV was markedly decreased. Similarly, a previous clinical study noted decreases from $95.9 \%$ to $58.8 \%$ in treatment-naive patients, from $91.5 \%$ to $28 \%$ in previous non-responders, and from $90.1 \%$ to $36.7 \%$ in ineligible/intolerant patients. ${ }^{14}$ This study also revealed similar results, although there were fewer RAV positive patients. RAV examinations in Korea are done at the Seoul Medical Science Institute using direct sequencing. If minor sequences of RAV are detected in more than $10 \%$ of the major sequence, the assigned result is RAV positive. ${ }^{15}$ If the RAV including $\mathrm{L} 31$ and $Y 93$ is lower than 10\%, the result is negative. Whether a prevalence of RAV $<10 \%$ influences SVR is still unclear.

Another factor affecting SVR12 is pretreatment HCV RNA level. The HALLMARK DUAL study reported an odds ratio of SVR12 of 3.38 for HCV RNA $<800,000 \mathrm{lU} / \mathrm{mL}$. SVR12 was higher with lower HCV RNA in treatment-naïve ( $96 \%$ vs. $87 \%$ ) patients, previous non-responders ( $93 \%$ vs. $80 \%)$, and in ineligible/intolerant $(88 \%$ vs. $80 \%$ ) patients. ${ }^{14}$ However, presently patients with HCV RNA $\geq 6$ $\log _{10} \mathrm{IU} / \mathrm{mL}$ displayed higher SVR12 rate (90.5\% vs. $88.2 \%$ ). The present results should be interpreted cautiously, given the low numbers of patients in the groups.

DAA therapy including DCV+ASV is convenient and less often has adverse events compared to treatment involving peginterferon. The latter treatment can be stopped due to adverse events including myalgia, anemia, neutropenia, and depression, and has a reportedly low cure rate, especially in patients with cirrhosis. ${ }^{16,17}$ In contrast, DCV+ASV produce mild adverse events in most clinical studies including patients with cirrhosis. Furthermore, SVR12 has been shown to be unaffected by presence of cirrhosis. ${ }^{14,18,19}$ Presently, the SVR12 rate was lower in patients with cirrhosis. The dichotomy between prior and the present studies need clarification with more patients.

The most common adverse event of DCV+ASV is liver enzyme elevation; its prevalence is about $2 \% .^{14}$ In this study, the prevalence of liver enzyme elevation was 2.6\%. The American Association for the Study of Liver Disease and KASL guidelines recommend that drug treatment should be stopped when enzyme elevation is $\geq 10$ times. $^{2,3}$ There was no discontinued case in our study. Skin problems can developed in all DAA treatment regimens, with a prevalence of up to $9.8 \%$ in combination with ribavirin and $3.8 \%$ without ribavirin. ${ }^{20}$ Presently, four patients complained of skin problems and two stopped DCV+ASV treatment. It is very rare to stop DAA treatment due to skin problems. The 
patient who stopped the drug due to severe skin rash and itching achieved SVR12 even though medication was taken for only 4 months. This is similar with the patients who stopped the drug due to markedly elevated liver enzyme in previous clinical studies. This means there is a possibility to achieve SVR12 as long as medication is taken, even if the 24-week treatment regimen is not completed.

Up to 150 million individuals worldwide suffer from chronic HCV infection, with a prevalence of approximately $1 \%$ in Korea. ${ }^{8,21}$ Therefore, the development of DAA is good news to patients with chronic hepatitis C. We confirmed that DCV+ASV in Korean patients has similar treatment efficacy and adverse events reported in prior clinical studies. But close monitoring to virologic breakthrough and severe adverse events are still needed.

\section{Conflicts of Interest}

The authors have no conflicts to disclose.

\section{REFERENCES}

1. Webster DP, Klenerman P, Dusheiko GM. Hepatitis C. Lancet 2015;385:1124-1135.

2. AASLD/IDSA HCV Guidance Panel. Hepatitis C guidance: AASLDIDSA recommendations for testing, managing, and treating adults infected with hepatitis C virus. Hepatology 2015;62:932-954.

3. Korean Association for the Study of the Liver. KASL clinical practice guidelines: management of hepatitis C. Clin Mol Hepatol 2016;22:76-139.

4. European Association for Study of Liver. EASL recommendations on treatment of hepatitis C 2015. J Hepatol 2015;63:199-236.

5. Gao M. Antiviral activity and resistance of HCV NS5A replication complex inhibitors. Curr Opin Virol 2013;3:514-520.

6. McPhee F, Sheaffer AK, Friborg J, Hernandez D, Falk P, Zhai G, et al. Preclinical profile and characterization of the hepatitis C virus NS3 protease inhibitor asunaprevir (BMS-650032). Antimicrob Agents Chemother 2012;56:5387-5396.

7. Lok AS, Gardiner DF, Lawitz E, Martorell C, Everson GT, Ghalib R, et al. Preliminary study of two antiviral agents for hepatitis $C$ genotype 1. N Engl J Med 2012;366:216-224.

8. Seong MH, Kil H, Kim YS, Bae SH, Lee YJ, Lee HC, et al. Clinical and epidemiological features of hepatitis $C$ virus infection in South Korea: a prospective, multicenter cohort study. J Med Virol 2013;85:1724-1733.

9. Kao JH, Lee YJ, Heo J, Ahn SH, Lim YS, Peng CY, et al. All-oral daclatasvir plus asunaprevir for chronic hepatitis $\mathrm{C}$ virus (HCV) genotype 1b infection: a sub-analysis in Asian patients from the HALLMARK DUAL study. Liver Int 2016;36:1433-1441.

10. Kumada H, Suzuki Y, Ikeda K, Toyota J, Karino Y, Chayama K, et al. Daclatasvir plus asunaprevir for chronic HCV genotype $1 \mathrm{~b}$ infection. Hepatology 2014;59:2083-2091.

11. Bartels DJ, Sullivan JC, Zhang EZ, Tigges AM, Dorrian JL, De Meyer S, et al. Hepatitis $C$ virus variants with decreased sensitivity to directacting antivirals (DAAs) were rarely observed in DAA-naive patients prior to treatment. J Virol 2013;87:1544-1553.

12. Karino Y, Toyota J, Ikeda K, Suzuki F, Chayama K, Kawakami Y, et al. Characterization of virologic escape in hepatitis $C$ virus genotype $1 \mathrm{~b}$ patients treated with the direct-acting antivirals daclatasvir and asunaprevir. J Hepatol 2013;58:646-654.

13. Miura M, Maekawa S, Sato M, Komatsu N, Tatsumi A, Takano S, et al. Deep sequencing analysis of variants resistant to the non-structural $5 \mathrm{~A}$ inhibitor daclatasvir in patients with genotype $1 \mathrm{~b}$ hepatitis C virus infection. Hepatol Res 2014;44:E360-E367.

14. Manns M, Pol S, Jacobson IM, Marcellin P, Gordon SC, Peng CY, et al; HALLMARK-DUAL Study Team. All-oral daclatasvir plus asunaprevir for hepatitis C virus genotype $1 \mathrm{~b}$ : a multinational, phase 3 , multicohort study. Lancet 2014;384:1597-1605.

15. Itakura J, Kurosaki M, Takada H, Nakakuki N, Matsuda S, Gondou $K$, et al. Naturally occurring, resistance-associated hepatitis $C$ virus NS5A variants are linked to interleukin-28B genotype and are sensitive to interferon-based therapy. Hepatol Res 2015;45:E115-E121.

16. Bruno S, Shiffman ML, Roberts SK, Gane EJ, Messinger D, Hadziyannis $S J$, et al. Efficacy and safety of peginterferon alfa-2a (40KD) plus ribavirin in hepatitis $C$ patients with advanced fibrosis and cirrhosis. Hepatology 2010;51:388-397.

17. Bourlière M, Khaloun A, Wartelle-Bladou C, Oules V, Portal I, Benali $S$, et al. Future treatment of patients with HCV cirrhosis. Liver Int 2012;32(Suppl 1):113-119.

18. Muir AJ, Poordad F, Lalezari J, Everson G, Dore GJ, Herring R, et al. Daclatasvir in combination with asunaprevir and beclabuvir for hepatitis C virus genotype 1 infection with compensated cirrhosis. JAMA 2015;313:1736-1744.

19. Nam HC, Lee HL, Yang H, Song MJ. Efficacy and safety of daclatasvir and asunaprevir for hepatitis C virus genotype $1 \mathrm{~b}$ infection. Clin Mol Hepatol 2016;22:259-266.

20. Patel P, Malik K, Krishnamurthy K. Cutaneous adverse events in chronic hepatitis $C$ patients treated with new direct-acting antivirals: a systematic review and meta-analysis. J Cutan Med Surg 2016;20:58-66.

21. World Health Organization (WHO). Fact sheet $N^{\circ} 164$ : hepatitis C. WHO web site, <http://www.who.int/mediacentre/factsheets/ fs164_apr2014/en/>. Accessed 2014.07.03. 